\title{
Validation of the procedure for the simultaneous determination of aflatoxins ochratoxin $A$ and zearalenone in cereals using HPLC-FLD
}

\begin{abstract}
Method validation for quantitative analysis of aflatoxins (AFs), ochratoxin A (OTA) and zearalenone (ZEA) in cereals using HPLC with fluorescence detector (FLD) is described. Mycotoxins were extracted with methanol : water $(80: 20)$ and purified with a multifunctional AOZ immunoaffinity column before HPLC analysis. The validation of the analytical method was performed to establish the following parameters: specificity, selectivity, linearity, limits of detection (LOD) and quantification (LOQ), accuracy, precision (within- and between-day variability), stability, robustness, measurement of performance, and measurement of uncertainty. Calibration curves were linear $(r>0.999)$ over the concentration range, from the LOQ to 26, 40 and $400 \mathrm{ng} / \mathrm{g}$ for AFs, OTA and ZEA, respectively. LOD and LOQ were 0.0125 and $0.05 \mathrm{ng} / \mathrm{g}$ for aflatoxin B1 (AFB1) and G1 (AFG1), 0.0037 and 0.015 $\mathrm{ng} / \mathrm{g}$ for aflatoxin B2 (AFB2) and G2 (AFG2), as well as 0.05 and $0.2 \mathrm{ng} / \mathrm{g}$ for OTA and 0.5 and $2 \mathrm{ng} / \mathrm{g}$ for ZEA, respectively. The mean recovery values were $77-104 \%$ for different concentrations of AFs, OTA and ZEA in spiked cereal samples. Both intra- and inter-day accuracy and precision were within acceptable limits. This method was successfully applied for the simultaneous determination of mycotoxins for 60 cereal samples collected from Malaysian markets. Fifty per cent of the cereal samples were contaminated with at least one of these mycotoxins, at a level greater than the LOD. Only one wheat sample and two rice samples were contaminated with levels greater than the European Union regulatory limits for AFs and OTA ( 4 and $5 \mathrm{ng} / \mathrm{g}$ ). The means and ranges of mycotoxins obtained for the cereal samples were $0.4 \mathrm{ng} / \mathrm{g}$ and $0.01-5.9 \mathrm{ng} / \mathrm{g}$ for total AFs; $0.18 \mathrm{ng} / \mathrm{g}$ and $0.03-5.3 \mathrm{ng} / \mathrm{g}$ for OTA; and $2.8 \mathrm{ng} / \mathrm{g}$ and $2.4-73.1 \mathrm{ng} / \mathrm{g}$ for ZEA, respectively. The results indicate that the method is suitable for the simultaneous determination of AFs, OTA and ZEA in cereals and is suitable for routine analysis.
\end{abstract}

Keyword: high-performance liquid chromatography (HPLC), mycotoxins, aflatoxins, ochratoxin A, zearalenone, cereals 